\title{
Structure and Optical Properties of ZnO Nanowires Fabricated by Pulsed Laser Deposition on GaN/Si(111) Films with the Use of Au and NiO Catalysts
}

\author{
E. M. Kaidashev ${ }^{a, e}$, M. Lorenz ${ }^{b}$, J. Lenzner ${ }^{b}$, A. Ramm ${ }^{b}$, T. Nobis ${ }^{b}$, M. Grundmann ${ }^{b}$, \\ N. Zakharov ${ }^{c}$, A. T. Kozakov ${ }^{d}$, S. I. Shevtsova ${ }^{d}$, K. G. Abdulvakhidov ${ }^{e}$, and V. E. Kaidashev ${ }^{e}$ \\ ${ }^{a}$ Mechanics and Applied Mathematics Research Institute, Southern State University, Rostov-on-Don, 344006 Russia \\ ${ }^{b}$ Leipzig University, Experimental Physics II Institute, Germany \\ ${ }^{c}$ MPI Microstructure Physics, Halle/Saale, Germany \\ ${ }^{d}$ Physics Research Institute, Southern State University, 344006 Russia \\ ${ }^{e}$ Southern State University, Rostov-on-Don, 344006 Russia \\ e-mail: Kaidashev@mail.ru
}

\begin{abstract}
The growth of $\mathrm{ZnO}$ nanowires on a $\mathrm{Si}(111)$ substrate with a $\mathrm{GaN}$ buffer layer by pulsed laser deposition at a high argon pressure and with the use of $\mathrm{Au}$ and $\mathrm{NiO}$ catalysts has been investigated. Application of the low-temperature $\mathrm{NiO}$ catalyst makes it possible to reduce the optimal growth temperature from $900^{\circ} \mathrm{C}$ (in the case of the Au catalyst) to $T=570^{\circ} \mathrm{C}$ and grow $\mathrm{ZnO}$ nanowires $20 \mathrm{~nm}$ in diameter, which are highly-oriented in the direction of the $c$ lattice axis and completely free of dislocations.
\end{abstract}

DOI: $10.3103 / \mathrm{S} 1062873808080315$

A promising direction in nanotechnology is the development of methods of self-assembled growth of semiconductor nanowires. The combination of high optical, mechanical, and piezoelectric properties of $\mathrm{ZnO}$ determines good prospects of this material in design of new nanoelectronic devices.

Hybrid structures based on $\mathrm{ZnO}$ nanowires and films that are highly-oriented along the $c$ axis are promising as basic elements of $\mathrm{UV} \mathrm{ZnO}$ nanolasers with optical, electrical, and electron-beam pumping, UV photodetectors, nanotransistors, electron emitters, piezoelectric nanocomposites with a dielectric or polymer filling, piezoelectric transducers and acoustic receivers, high-efficiency solar energy converters, nanosensors of chemical and biological materials, and elements of devices for nanomechanics and nanospintronics.

Despite significant efforts, $p$ - $\mathrm{ZnO}$ structures with high mobility have not yet been obtained. Therefore, it was proposed in [1] to use $n-\mathrm{ZnO} / p$ - GaN for heterojunctions to fabricate light-emitting $\mathrm{ZnO}$-based film structures. The first light-emitting heterostructure of the $n$ - $\mathrm{ZnO}$ nanowire/epitaxial $p$-GaN film type was investigated in [2]. However, the low electroluminescence intensity in the UV region and high-intensity green luminescence indicated poor structural quality of the nanostructures obtained.

We investigated the growth of $\mathrm{ZnO}$ nanowires on an $n$-GaN epitaxial film formed on a (111)Si substrate by organometallic vapor deposition. $\mathrm{ZnO}$ nanowires were fabricated by pulsed laser deposition [3-6] in an evacu- ated quartz cell using an external resistive heater. Radiation of a $\mathrm{KrF}$ laser $(\lambda=248 \mathrm{~nm})$ was focused on the surface of a rotating $\mathrm{ZnO}$ ceramic target. The energy density in a pulse on the target surface was $2 \mathrm{~J} \mathrm{~cm}^{-2}$. The laser pulse repetition rate was 3-10 Hz. Synthesis of $\mathrm{ZnO}$ nanowires was performed applying from 12 to 24 thousand laser pulses. The substrate temperature was varied in the range $850-950^{\circ} \mathrm{C}$. The quartz cell was previously evacuated to a pressure of $5 \mathrm{~Pa}$. Then highpurity $(99.998 \%)$ argon was leeked into the chamber. The argon flow was $0.21 \mathrm{~min}^{-1}$ at a pressure of 75 200 mbar. Si(111) substrates with previously deposited epitaxial GaN films were oriented parallel to the laser torch at a distance of $20-35 \mathrm{~mm}$ from the target surface. A thin gold layer $(h=2-3 \mathrm{~nm})$, used as a catalyst, was deposited on the substrate by rf magnetron sputtering.

As our experiments showed, at temperatures from 900 to $1100^{\circ} \mathrm{C}$, an Au film with a thickness of $3-5 \mathrm{~nm}$ is divided into isolated nanoislands due to the self-organization. With an increase in the film thickness, the average and maximum sizes of the nanoislands increase, as well as the spread of their diameters. An increase in temperature from $900-1100^{\circ} \mathrm{C}$ increases the average and maximum sizes of nanoislands and decreases their density. The most uniform distribution of nanoislands with approximately equal diameters was observed for a (3-5)-nm-thick gold film, previously heated to $900^{\circ} \mathrm{C}$ in an argon atmosphere. 


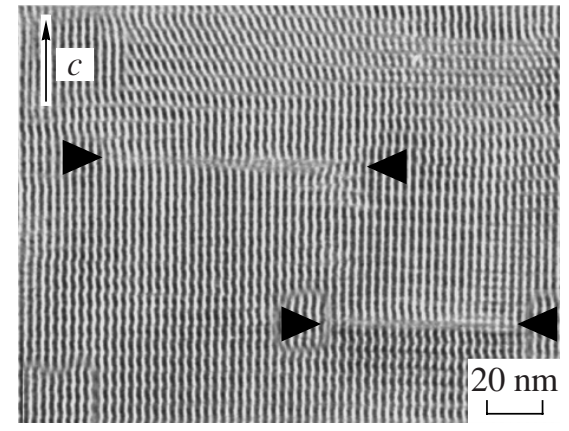

Fig. 1. HRTEM image $(400 \mathrm{kV})$ of the growth defects in $\mathrm{ZnO}$ nanowires on a $\mathrm{Si}(111)$ substrate with a $\mathrm{GaN}$ film ( $\mathrm{Au}$ catalyst, growth temperature $900^{\circ} \mathrm{C}$ ).

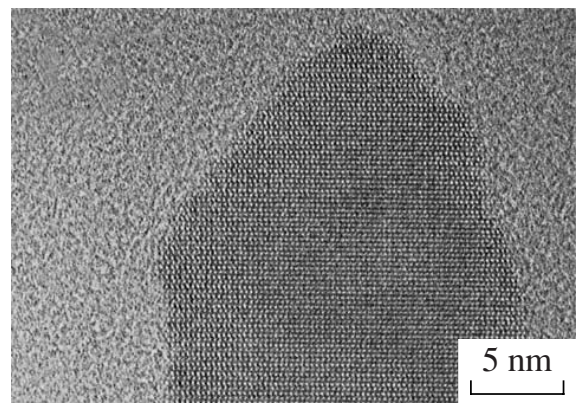

Fig. 2. HRTEM image $(400 \mathrm{kV})$ of the top part of an isolated $\mathrm{ZnO}$ nanowire on $\mathrm{NiO} / \mathrm{GaN} / \mathrm{Si}(111)$.
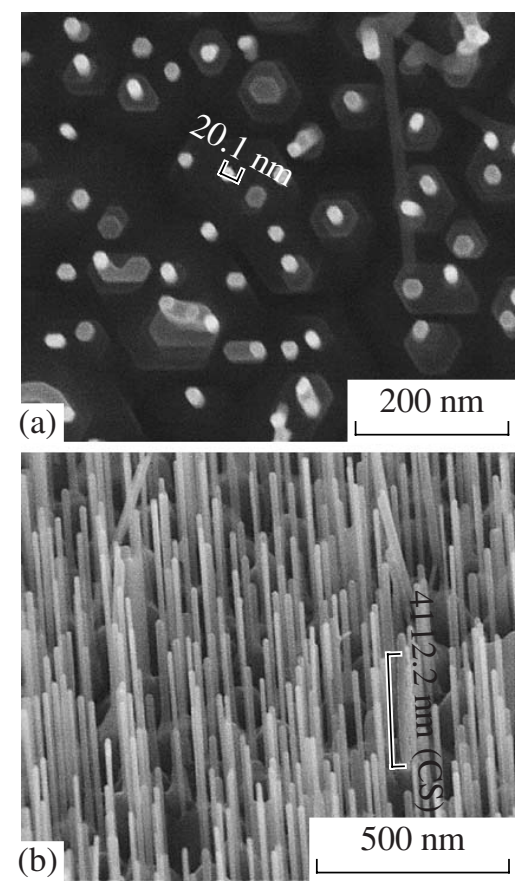

Fig. 3. Secondary-electron image of the surface of $\mathrm{ZnO}$ nanowires $20 \mathrm{~nm}$ in diameter, grown on a $\mathrm{Si}(111)$ substrate with an epitaxial $\mathrm{GaN}$ layer (catalyst $\mathrm{NiO}$ ). The observation angles are (a) $0^{\circ}$ and (b) $45^{\circ}$.
The surface morphology of the nanowires was investigated on a CamScan CS44 scanning electron microscope and a Comebax-micro electron microscope microanalyzer. According to the measurements, when gold was used as a catalyst, the nanowires were $65 \mathrm{~nm}$ in diameter and 1-2 $\mu \mathrm{m}$ in height for the growth at $900^{\circ} \mathrm{C}$ in $\operatorname{argon}$. In the initial growth stages, drops of the catalyst (gold) are observed on the nanowire tips. An epitaxial $\mathrm{ZnO}$ film, formed in the initial growth stage, was observed between the GaN film and $\mathrm{ZnO}$ nanowire. Investigation by high-resolution transmission electron microscopy (HRTEM) showed that the high growth temperature $\left(900^{\circ} \mathrm{C}\right)$ (characteristic of the use of a thin gold film as a catalyst) facilitates the formation of misfit dislocations in $\mathrm{ZnO}$ nanowires in directions perpendicular to the $c$ axis in the early growth stage (Fig. 1).

The microphotoluminescence spectra of the lattice of $\mathrm{ZnO}$ nanowires grown on a $\mathrm{Si}(111)$ substrate with a GaN buffer film using gold as a catalyst showed a high photoluminescence intensity at room temperature in the UV range and a very weak peak in the visible range, which is indicative of low concentration of oxygen vacancies in the nanowires grown. The width of the emission peak of the edge exciton $D^{0} X$ was $1.7 \mathrm{meV}$ $(T=2 \mathrm{~K})$; it coincides with the corresponding value for high-quality epitaxial $\mathrm{ZnO}$ films obtained by us by multistep pulsed laser deposition [7].

The structure of the films was investigated on a Phillips X'Pert high-resolution X-ray diffractometer. The analysis showed that the X-ray diffraction patterns obtained by $2 \theta-\omega$ scanning of the $\mathrm{ZnO}$ nanowire lattice contain only 002 and 004 reflections of $\mathrm{ZnO}$ nanowires; this fact indicates that the $c$ axis of $\mathrm{ZnO}$ nanowires is oriented perpendicularly to the substrate and coincides with the preferred growth direction. The half-width of the $\mathrm{ZnO} 0002$ reflection in the $\theta-2 \theta$ scanning mode was $33 \mathrm{arcsec}$, and the half-width of the rocking curve of the 0002 reflection was $\Omega=1564$ arcsec. For comparison, the half-width of the $\mathrm{ZnO} 0002$ reflection in the $\theta-2 \theta$ scanning mode is 131 arcsec for the high-quality epitaxial $\mathrm{ZnO}$ film, obtained by us using pulsed laser deposition at a low oxygen pressure [7].

To decrease the optimal growth temperature of $\mathrm{ZnO}$ nanowires, we investigated their growth on a $\mathrm{GaN} / \mathrm{Si}(111)$ substrate using a thin (3-4 nm) NiO film as a catalyst. The $\mathrm{NiO}$ film was formed by pulsed laser deposition. The use of a low-temperature $\mathrm{NiO}$ catalyst (at the same pressures and flow velocities of argon and target-substrate distances) makes it possible to reduce the growth temperature to $570^{\circ} \mathrm{C}$. The minimum radius of a growing nanowire decreases (at a constant gas flow), approximately inversely proportionally to the growth temperature [8]. HRTEM analysis showed that $\mathrm{ZnO}$ nanowires in the $\mathrm{ZnO} / \mathrm{NiO} / \mathrm{GaN} / \mathrm{Si}(111)$ structure are completely free of dislocations and have a perfect internal structure (Fig. 2). The nanowires grown at the 
substrate temperature $T=570^{\circ} \mathrm{C}$ have a diameter of 20 $\mathrm{nm}$ and a surface density of $10^{9} \mathrm{~cm}^{-2}$ (Fig. 3).

In this study, we have implemented growth of $\mathrm{ZnO}$ nanowires, highly-oriented along the $c$ crystallographic axis, on GaN films by pulsed laser deposition at a high argon pressure, using $\mathrm{Au}$ and $\mathrm{NiO}$ catalysts. In contrast to the nanowires grown at a high temperature, the lowtemperature $\mathrm{ZnO}$ nanowires grown on an ultrathin $\mathrm{NiO}$ catalyst film have a diameter up to $20 \mathrm{~nm}$, are almost completely free of dislocations, and possess a perfect internal structure.

The high structural and optical properties of the obtained $\mathrm{ZnO}$ nanowire/GaN film hybrid heterostructures on a silicon substrate make them promising for application in new devices of nanoelectronics and nanophotonics.

\section{REFERENCES}

1. Alivov, Ya.I., van Nostrand, J.E., Look, M.D., et al., Appl. Phys. Lett., 2003, vol. 83, p. 2943.

2. Park, B.W. and Yi, G.C., Adv. Mater., 2004, no. 16, p. 87.

3. Lorenz, M., Kaidashev, E.M., Rahm, A., et al., Appl. Phys. Lett., 2005, vol. 86, 143113.

4. Nobis, T., Kaidashev, E.M., Rahm, A., et al., Nano Lett., 2004, no. 4, p. 797.

5. Nobis, T., Kaidashev, E.M., Rahm, A., et al., Phys. Rev. Lett., 2004, vol. 93, no. 10, 1039031.

6. Lorenz, M., Lenzner, J., Kaidashev, E.M., and Hochmuth, H., Ann. Phys., 2004, vol. 2, no. 1, p. 39.

7. Kaidashev, E.M., Lorenz, M., Wenckstern, H., et al., Appl. Phys. Lett., 2003, vol. 82, p. 3901.

8. Dubrovskii, V.G. and Sibirev, N.V., Phys. Rev. E: Stat., Nonlinear, Soft Matter Phys., 2004, vol. 70, 031604. 